\title{
SUBPHRENIC ABSCESS
}

\author{
BY \\ GEOFFREY H. WOOLER \\ Leeds
}

(RECEIVED FOR PUBLICATION JANUARY 2, 1956)

The term subphrenic abscess denotes infection occurring in the region bounded by the diaphragm above, and by the transverse meso-colon, transverse colon, and great omentum, below. The liver divides this region into upper and lower compartments. The suprahepatic compartment is divided into left and right portions by the falciform ligament, and the infrahepat:c compartment is similarly divided by the ligamentum teres and the ligamentum venosum. Infection in these peritoneal spaces or in the retroperitoneal tissues immediately behind them may be described as being subphrenic.

\section{ANATOMY}

In 1907 Barnard read a paper at the Royal Society of Medicine which he called "The Surgical Aspects of Subphrenic Abscess." $\mathrm{He}$ began by describing the peritoneal spaces botween the diaphragm and the liver, and stated that both left and right compartments were divided into anterior and posterior portions by the triangular and coronary ligaments of the liver. His description was simple, easy to memorize, and still appears in many of the surgical textbooks, but it is not correct anatomically.

It is rather difficult to visualize the size and positions of the two triangular ligaments of the liver from the descriptions which I have read. So in several post-mortem subjects lead clips were placed on the two triangular ligaments of the liver. Antero-posterior and both lateral radiographs were taken of the upper abdomen to show the relative size and position of these ligaments (Figs. 1 and 2). The subphrenic spaces were then packed with cotton wool soaked in barium emulsion and more radiographs taken to show the extent of these spaces.

The left triangular ligament is situated medially, almost in the centre of the abdomen. It lies more anteriorly than the right triangular and coronary ligaments and is a superior attachment of the liver. It is much stronger than any of the other ligaments and appears to be the main support of the liver.
The coronary ligament surrounds the bare area of the liver behind the right lobe. The right triangular ligament is the extreme right edge of this ligament.

The right triangular and coronary ligaments extend almost to the lateral abdominal wall and form a posterior attachment of the liver. Their two layers of peritoneum are reflected from the bare area of the liver and are therefore separated. Following Barnard's teaching many surgeons to-day believe that between the right lobe of the liver and the diaphragm there is an anterior and a posterior subphrenic space separated by the right triangular ligament. Ochsner and De Bakey (1938) and Thorek (1947) believe this is true, whereas Mitchell (1940) and Harley (1949) deny the existence of a right posterior subphrenic space above the liver.

The suprahepatic space lying between the right lobe of the liver and the diaphragm, bounded posteriorly by the coronary and right triangular ligaments, is very large (Figs. 3 and 4). It extends medially to the falciform ligament and laterally it communicates with the right paracolic gutter and Rutherford Morison's pouch. At operation it is not usual to find the whole of this space infected, but more commonly pus localizes either anteriorly or posteriorly in it. An anterior abscess (Fig. 5) may be drained through a subcostal incision, but laparotomy may fail to disclose a posterior one in this space which can only be reached transpleurally (Fig. 6). Thus on the right side there is a large suprahepatic space in front of the right triangular ligament. Below this ligament there is a smaller infrahepatic space which is really part of Rutherford Morison's pouch, and not of sufficient size to justify separation from it. Behind the peritoneum there is a retroperitoneal space which is also perinephric.

On the left side a large space separates the left lobe of the liver, the spleen, and the stomach from the diaphragm (Figs. 9 and 10). The left triangular ligament of the liver is narrow; it lies near the centre of the abdomen and is not sufficiently broad to divide this space into anterior and posterior 


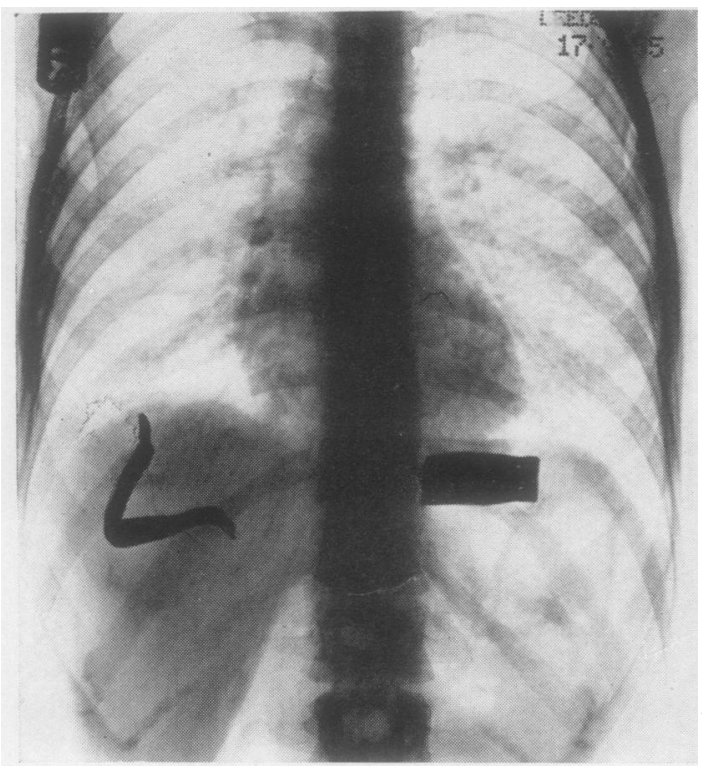

FIG. 1

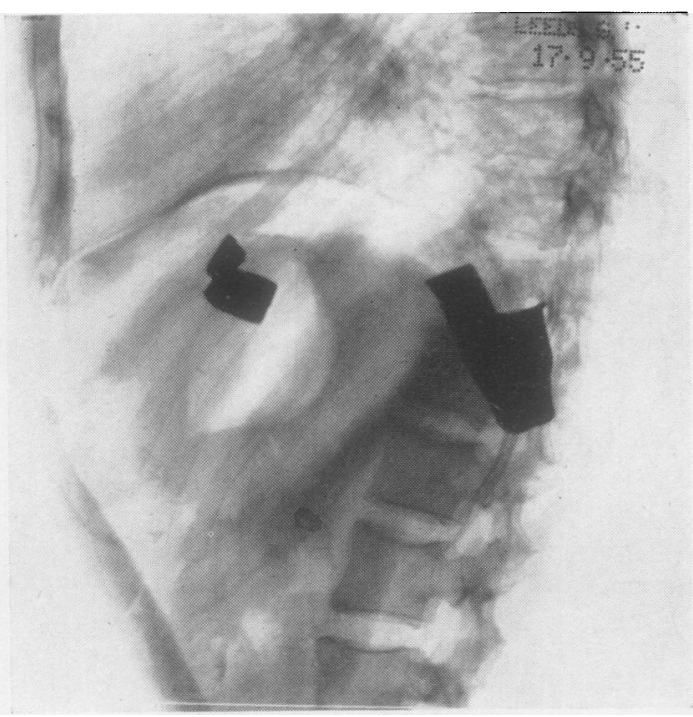

FIg. 2

FIGS. 1 and 2.-Lead clips have been placed on the left and right triangular ligaments of the liver. The left triangular ligament lies near to the mid line and is a superior attachment of the liver. The right triangular extends more laterally and lies at the back of the right lobe. Antero-posterior and lateral radiographs of the abdomen.

FIGs. 3 and 4.-The right suprahepatic subphrenic space has been packed with cotton wool soaked in barium emulsion. It is very large, and the lateral view shows that it extended from the front to the back of the under surface of the diaphragm.

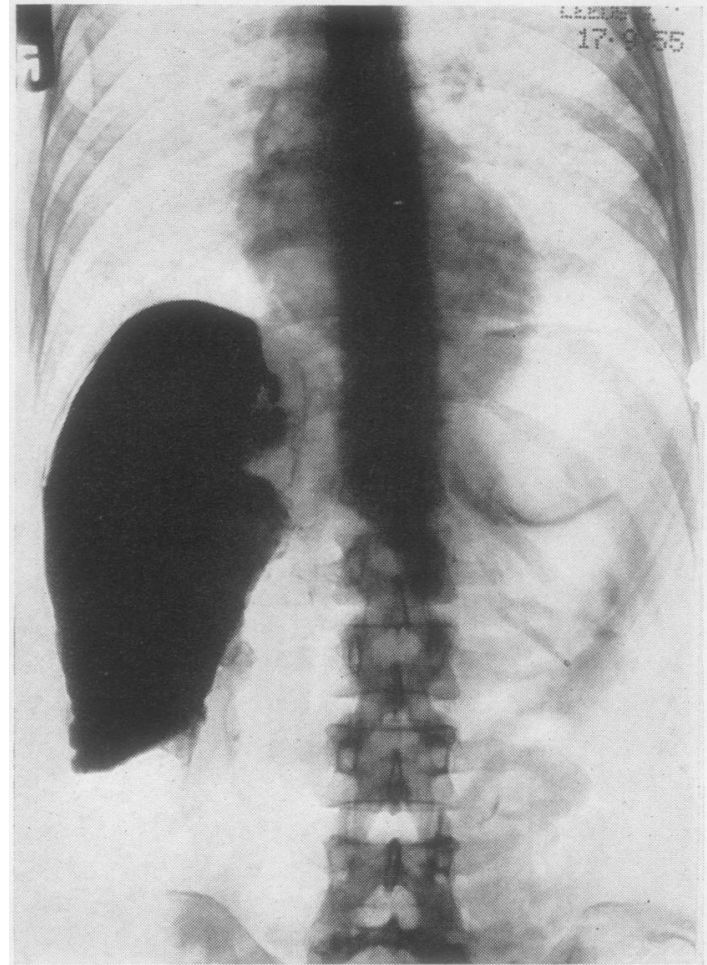

FIG. 3

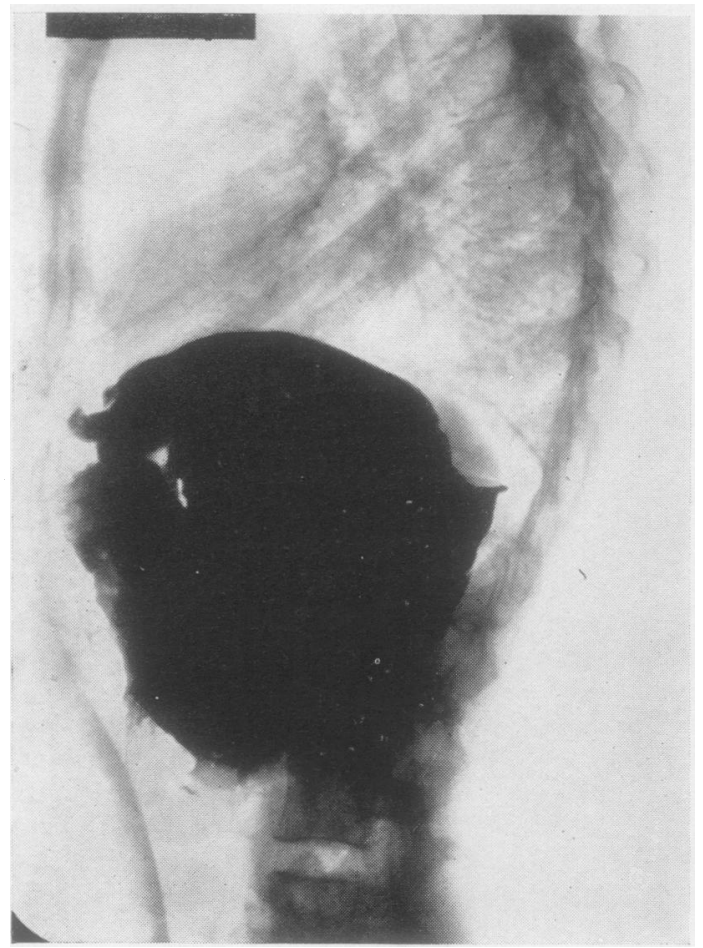

Fio. 4 


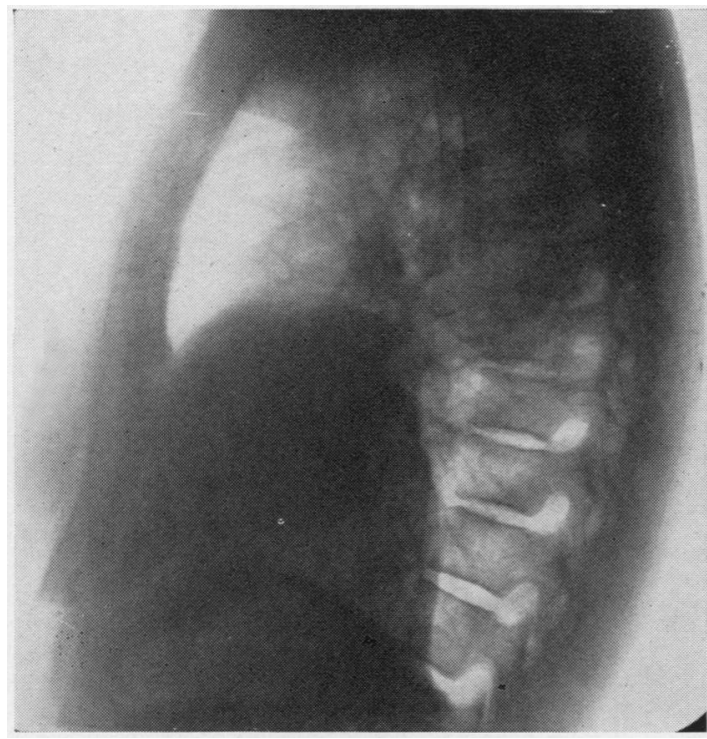

Fio. 5

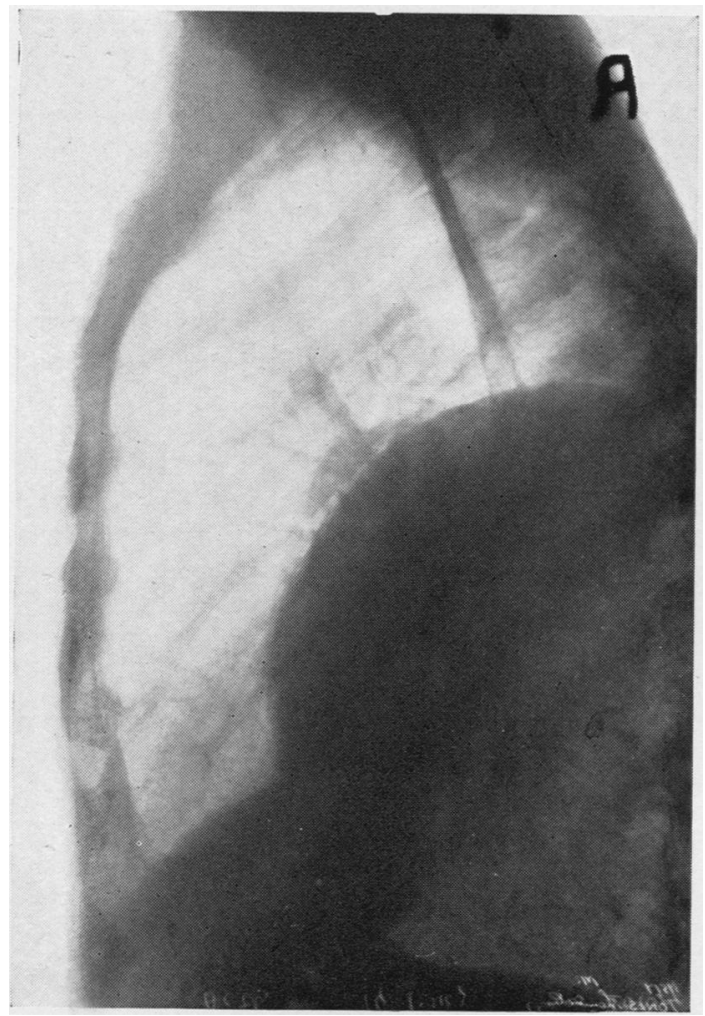

F10. 6

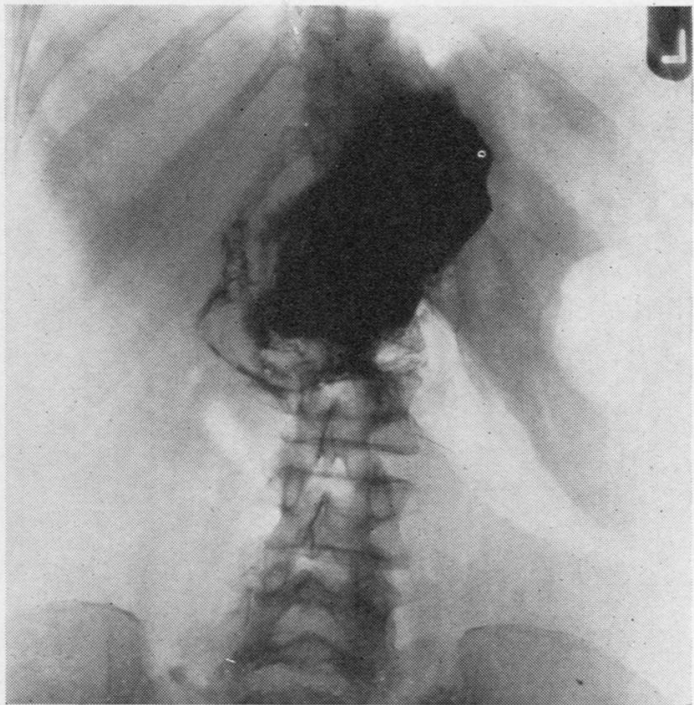

FIg. 7

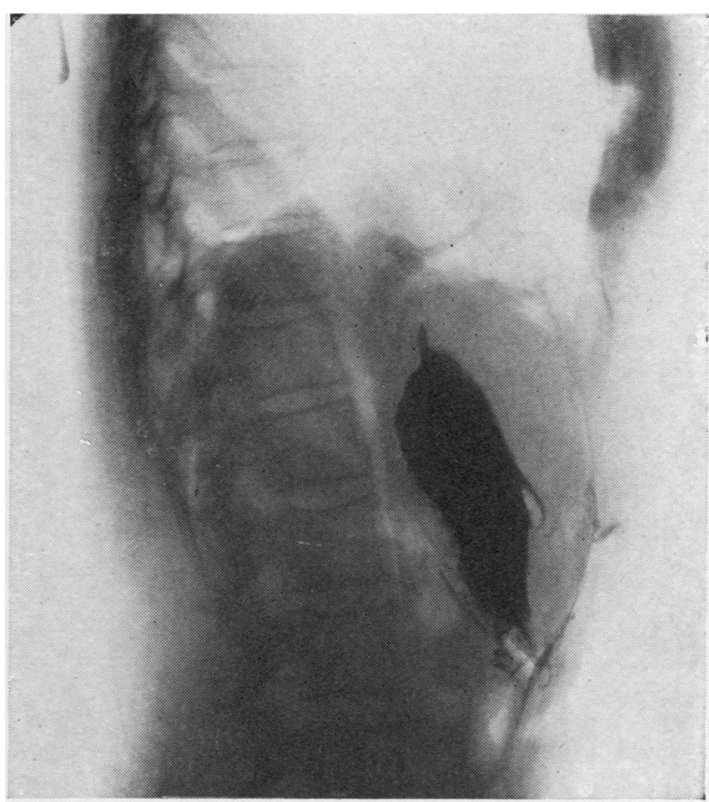

Fig. 8

Fig. 5.-An antericr atscess in the right supraheratic subphrenic space.

Fig. 6.-A posterior abscess in the right suprahepatic subphrenic space. This abscess is anterior to the right triangular ligament and above it.

Figs. 7 and 8.-The anterior infrahepatic subphrenic space on the left side is packed with cotton wool soaked in barium emulsion. It lies between the under surface of the left lobe of the liver and the stomach, and it is really an extension of the left suprahepatic subphrenic space. 

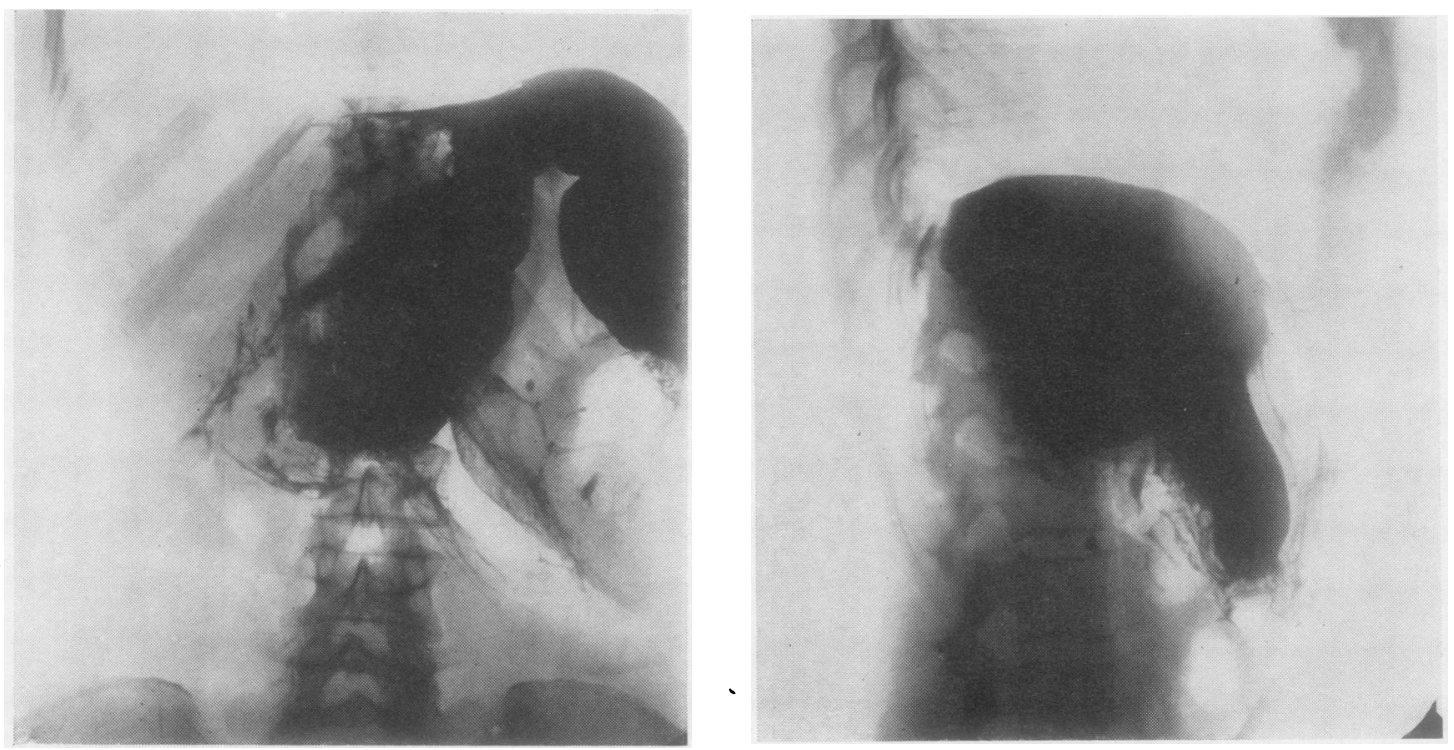

FIGs. 9 and 10.-The left suprahepatic space and the anterior infrahepatic space on the left side, both packed with cotton wool and barium emulsion. Notice that the stomach lies between these two spaces, and that the greater portion of the left suprahepatic space lies between the diaphragm and the fundus of the stomach.

portions as described by Barnard (1908). There is an extension of this space anteriorly under the left lobe of the liver between it and the stomach (Figs. 7 and 8): although normally it communicates widely with the large space under the left dome of the diaphragm, pus not infrequently localizes in this so-called extension forming another type of subphrenic abscess. Harley (1949) calls it the left anterior infrahepatic space, which describes its position fairly accurately.

The posterior infrahepatic space on the left side is the lesser sac lying behind the liver and the stomach, and there is also a retroperitoneal space above the left kidney.

\section{Aetiology}

During the past 10 years in the Department of Thoracic Surgery at Leeds, 50 patients with subphrenic abscess have been treated, the abscess being on the right side in 37 , on the left in 11 , and bilateral in two patients.

On the right side 10 followed a perforated peptic ulcer, five appendicectomy, five cholecystectomy, and three partial gastrectomy. Four were of insidious onset, and no obvious cause was found. Four were associated with infected hydatid cysts of the liver, two with perinephric abscess, one with a staphylococcal liver abscess, and another with an infected amoebic abscess of the liver. One was caused by a duodenal diverticulum perforating retroperitoneally, and the last followed hysterectomy and a pelvic abscess.

On the left side four followed gastric resections, two perforated peptic ulcers, and two appendicectomy. Two were associated with perinephric abscess and one with infected hydatid cysts of the liver and spleen.

One of the patients with bilateral subphrenic abscesses had recently had an appendicectomy, the other had an enormous infected amoebic abscess of the liver.

Only one of these 50 patients died. He was a man aged 72 who had both a right subphrenic abscess and an empyema. Both were drained, and the subphrenic abscess soon healed. The empyema cavity, however, remained as large as ever because the lung was held down by pleural thickening. A difficult decortication of the lung was performed at a later date and the patient died the following day.

All the others survived and were discharged with the sinuses healed.

\section{Diagnosis}

The history itself nearly always suggests that something is wrong between the abdomen and the chest on one side or the other. Thus 33 of our 50 patients had had an abdominal operation or catastrophe of some kind. After this acute episode each appeared to be well for a period of time 
varying between a few days and a few months. Then they had a rigor or began to run a fever, and developed pain under one costal margin or in the shoulder. Shoulder-tip pain is common and sometimes severe. One patient described it as being so bad that he thought he had dislocated his shoulder joint.

On the other hand the onset may be insidious without a previous history of an abdominal complaint. The patient begins to lose weight, runs a slight fever and feels tired, but has no localizing signs suggesting subphrenic infection, while the white cell count may be normal. Such was the course of the disease in a patient aged 42 who developed a staphylococcal liver abscess. $\mathrm{He}$ remained well while he was on antibiotics, but as soon as they were discontinued he began to run a fever and felt run down. The diagnosis was made later when the infection spread through the diaphragm into the right pleural cavity, and indeed before this happened there was nothing to suggest that he had anything wrong under the right diaphragm.

Lastly the patient may present with the complications of the disease which will be described later, but briefly they are (1) pleurisy with effusion, (2) empyema, and (3) lung abscess and bronchial fistula. If the fistula communicates with the biliary tract or the duodenum, the sputum contains bile.

Only one patient presented with peritonitis caused by a subphrenic abscess bursting into the general peritoneal cavity. He was a man of 65 years with an infected amoebic abscess of the liver, which spread into both suprahepatic spaces and thence throughout the abdomen. This mode of spread is uncommon with ordinary pyogenic abscesses, for the general peritoneal cavity is well sealed off by adhesions.

\section{Radiological Features}

An attempt was made to confirm the diagnosis in all these patients by radiology. The main findings were :

(1) Loss of diaphragmatic movement and elevation of the diaphragm, which may affect only one portion rather than the whole of the diaphragm (Fig. 11). The right side of the diaphragm elevates more readily than the left, and in one patient it reached the level of the third rib anteriorly (Figs. 12 and 13). Pus forming between the right lobe of the liver and the diaphragm has either to push the liver down or the diaphragm up ; the latter is easier (Figs. 14 and 15).

On the left side, elevation of the diaphragm is not such a marked feature because the liver does

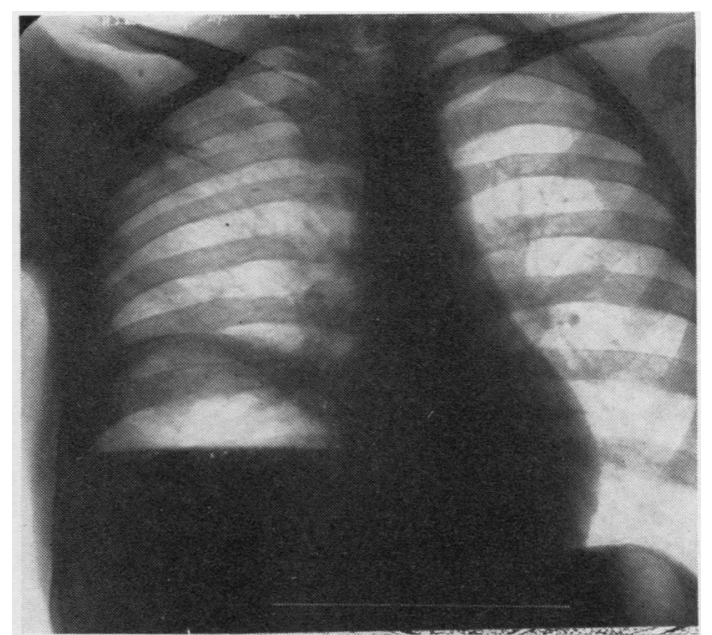

FIG. 11.-The right dome of the diaphragm was elevated, and the posterior half of it remained immobile on screening. A posterior suprahepatic subphrenic abscess, which contained over 2 pints of pus, was drained.

not extend to the lateral abdominal wall. The stomach nearly always forms part of the lower wall of the abscess and, being a fairly mobile structure, readily gives way as the abscess enlarges.

(2) The presence of gas or a fluid level under the diaphragm: it must be ascertained that this is not contained in a portion of the alimentary tract, and a barium meal or enema may be given if there is any doubt.

Loops of intestine are sometimes found between the right lobe of the liver and the diaphragm, but the intestinal markings can be seen, so they should present no difficulty. This is not an uncommon feature in Hirschsprung's disease, when the distension involves most of the large intestine, and again haustrations may be seen in the distended loops.

(3) Enlargement of the liver shadow.

(4) Thickening and a "woolly" appearance of the left dome of the diaphragm (Figs. 16, 17, and 18).

(5) The presence of a pleural effusion.

If screening the chest and the straight radiographs fail to reveal a suspected subphrenic abscess, a pneumoperitoneum may be induced. When a pneumoperitoneum has been induced and no air is shown in one subphrenic space (Fig. 19) this usually denotes infection, but if the space has been obliterated by adhesions no air will enter and so this method is not diagnostic.

Transpleural Diagnostic Aspiration

This should only be performed in the operating theatre so that if pus is obtained the abscess may 


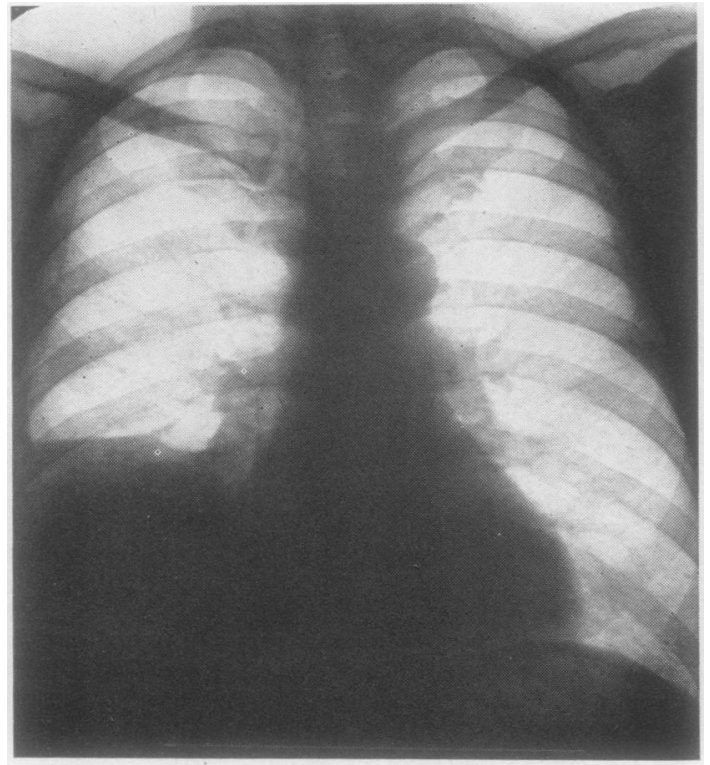

FIG. 12

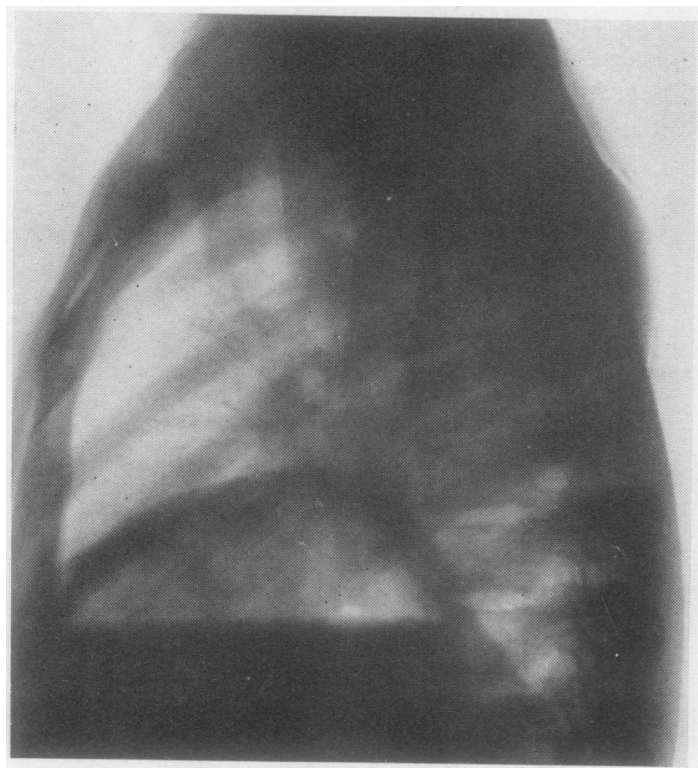

Fio. 13

FIGs. 12 and 13.-Large subphrenic abscess in a man aged 23, of insidious onset, but found to be due to perforation of a duodenal ulcer. The lateral view shows that it was an anterior right suprahepatic space infection. It was drained through a sutcostal incision.

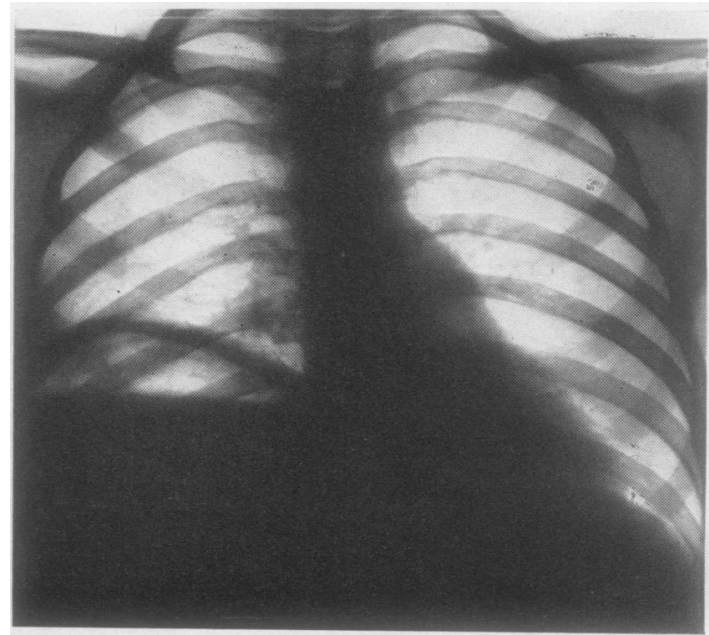

FIG. 14

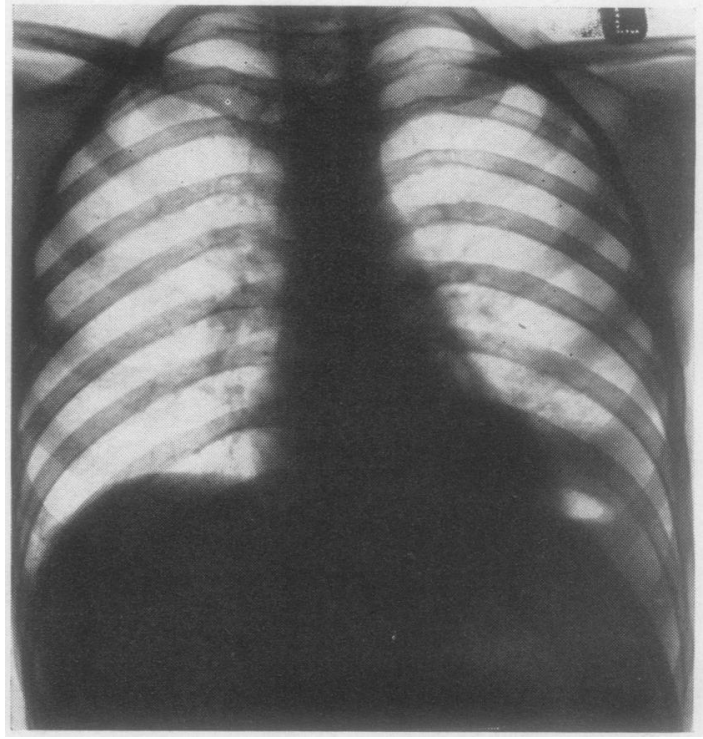

FIo. 15

FIGs. 14 (April, 1949) and 15 (June, 1949).-Bilateral subphrenic abscess following appendicectomy. The right one required drainage. The left one became apparent two months later when it ruptured into a bronchus, which resulted in a spontaneous cure. 


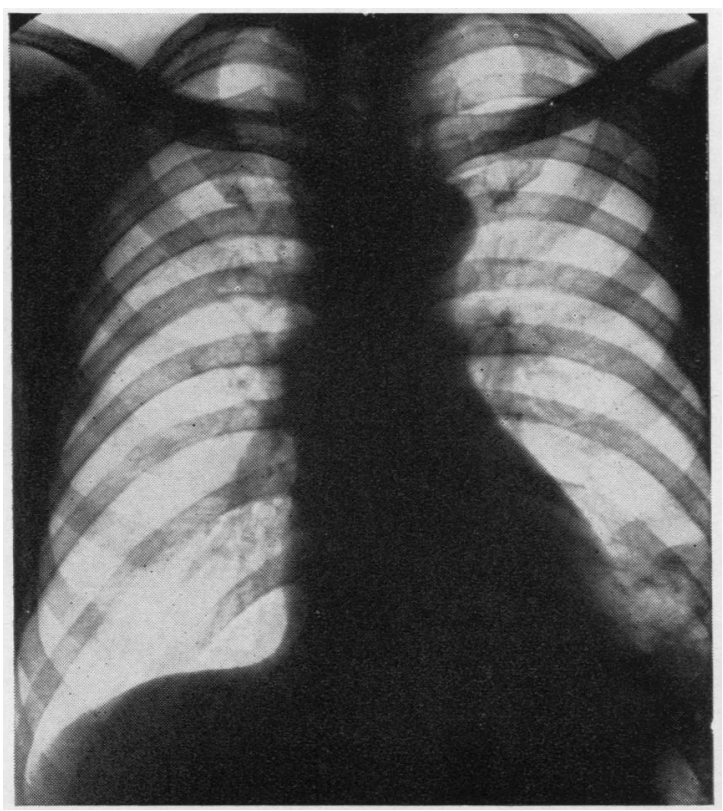

Fro. 16

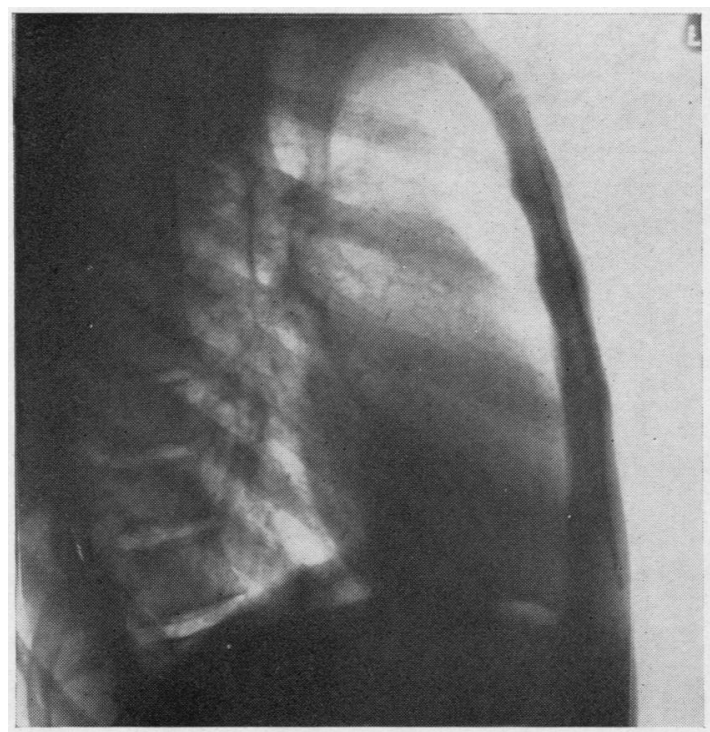

FIo. 17

FIGs. 16, 17 and 18.-A large chronic gastric ulcer which gradually perforated causing an insidious onset of a left subphrenic abscess in a man aged 50. The postero-anterior and lateral views of the chest show a fluid level under the left diaphragm and thickening of the left diaphragm.

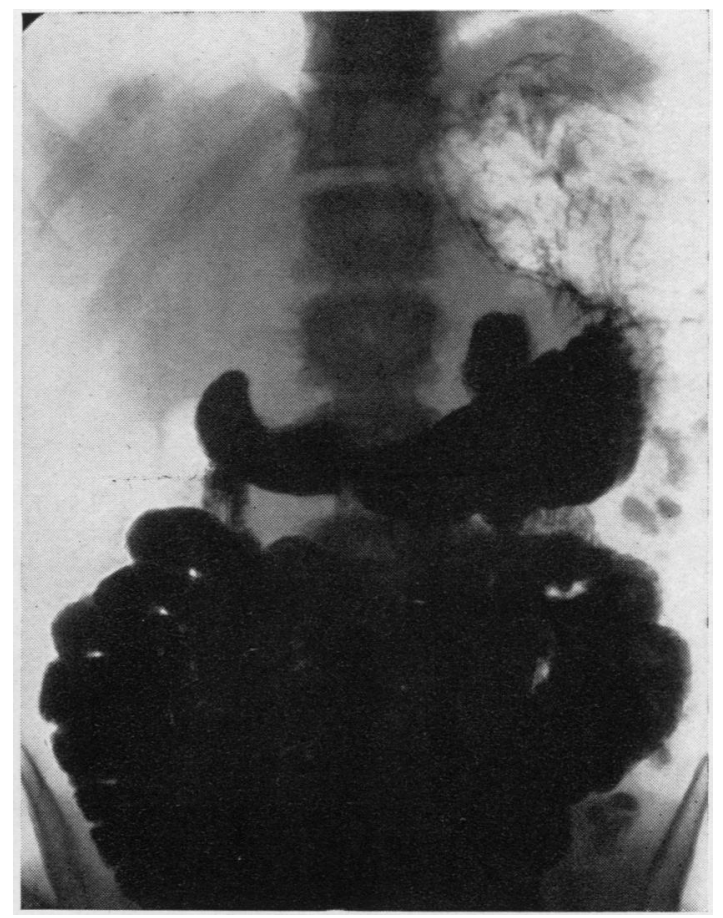

Fro. 18

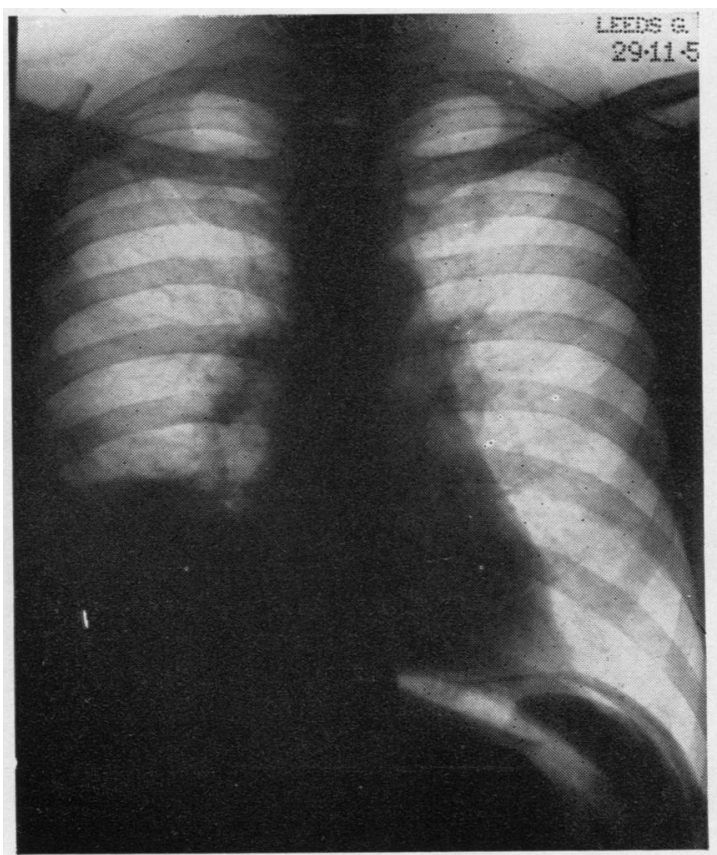

Fig. 19

Fig. 19.-A pneumoperitoneum showed a clear left diaphragm. There was a right subphrenic abscess caused by a staphylococcal liver abscess. 
be drained without delay. To delay proper drainage would result in pus leaking through the puncture hole in the diaphragm and produce an empyema.

\section{COMPLICATIONS}

The complications are mainly thoracic, for the infection frequently spreads through the diaphragm into the chest. It is rare for the infection to go down into the abdomen, involving the general peritoneal cavity and the pelvis.

The chest complications are:

Pleurisy.-Pleural pain is common and may be the main complaint, simulating a basal lobar pneumonia. The pain of diaphragmatic pleurisy is referred to the shoulder.

Pleural Effusion.-This may be sterile-of the so-called "sympathetic" type. It can be absorbed spontaneously after the abscess has been drained, or it may require aspiration.

EMPYEMA (Fig. 20).

LUNG ABSCESS, with bronchial fistula.

The inflammatory process in the chest begins with a diaphragmatic pleurisy. If fluid is formed, it pushes the lung away from the subphrenic abscess and so prevents it from bursting into the lung. More frequently the lung becomes adherent to the diaphragm, and the fluid or empyema collects at a higher level in the paravertebral sulcus (Fig. 21).

A lung adherent to the diaphragm allows a subphrenic abscess to erode directly into a bronchus and be coughed up.

If the abscess is large the patient may almost drown in pus, and when this occurs immediate drainage of the abscess is necessary in order to save life (Figs. 22 and 23).

On the other hand I have had at least one patient who coughed up a small subphrenic abscess which then healed spontaneously. I operated upon her later and could find no residual pus but only healing fibrous tissue (Fig. 15).

Another patient developed gangrene of the left lower lobe when a subphrenic abscess ruptured into it. The whole lobe became necrotic and just a bag of pus. It was resected at the same time as the subphrenic abscess was drained, and the patient survived.

A third patient presented with recurrent attacks of haemoptysis and a little mucopurulent sputum. The radiograph of the chest showed an opacity in the left lower lobe which was considered to simulate a neoplasm. At thoracotomy the lower lobe was firmly adherent to the diaphragm, and when it was mobilized a subphrenic abscess was found which had ruptured into the left lower lobe (Figs. 24 and 25).

Spread to other peritoneal spaces from an established subphrenic abscess may occur, but is not common. This may happen when the abscess is infrahepatic, and pus leaks down one paracolic gutter into the pelvis.

The only patient in this series with a suprahepatic abscess which leaked into the general peritoneal cavity, so that he presented with generalized peritonitis, was the one already mentioned who had an infected amoebic abscess of the liver and bilateral subphrenic abscesses, which burst into the abdomen.

\section{Conservative Treatment}

There is no doubt that some inflammatory processes in the subphrenic spaces resolve with antibiotic treatment. These may have been caused by a chemical reaction rather than bacterial infection, e.g., from the perforation of a peptic ulcer. Clinically and radiologically they present the same features as a subphrenic abscess, but the fluid formed is not pus and they clear up on conservative treatment alone. They must, however, be watched for several months before they are pronounced as cured, because it is not unusual for a patient after an abdominal catastrophe to return about a year later with a subphrenic abscess.

Restricted movement of one portion of the diaphragm is the most valuable radiological sign. When this is not present, and when there is reasonable doubt over the diagnosis, no harm will ensue by waiting, provided the general condition of the patient does not deteriorate and a course of the appropriate antibiotic is given. So, when dealing with a doubtful case, one must keep a careful watch radiologically for spread of the infection towards the chest during this period of observation. If the diaphragm becomes elevated, or is seen not to move properly, or if fluid appears in the pleural space, then it is probable that conservative treatment should be abandoned. At this stage it would be justifiable to explore the subphrenic region with a needle. Aspiration will not damage the lung provided it is performed low down with the needle pointing up towards the subphrenic spaces. The needle used must be the same as for a chest aspiration, a number 17 B.W.G. with a short point. The thickness of the diaphragm may be felt with the needle, and this information alone has been helpful several times in diagnosing a subphrenic abscess, even when pus was not withdrawn. Only the peripheral parts of the subphrenic spaces may 


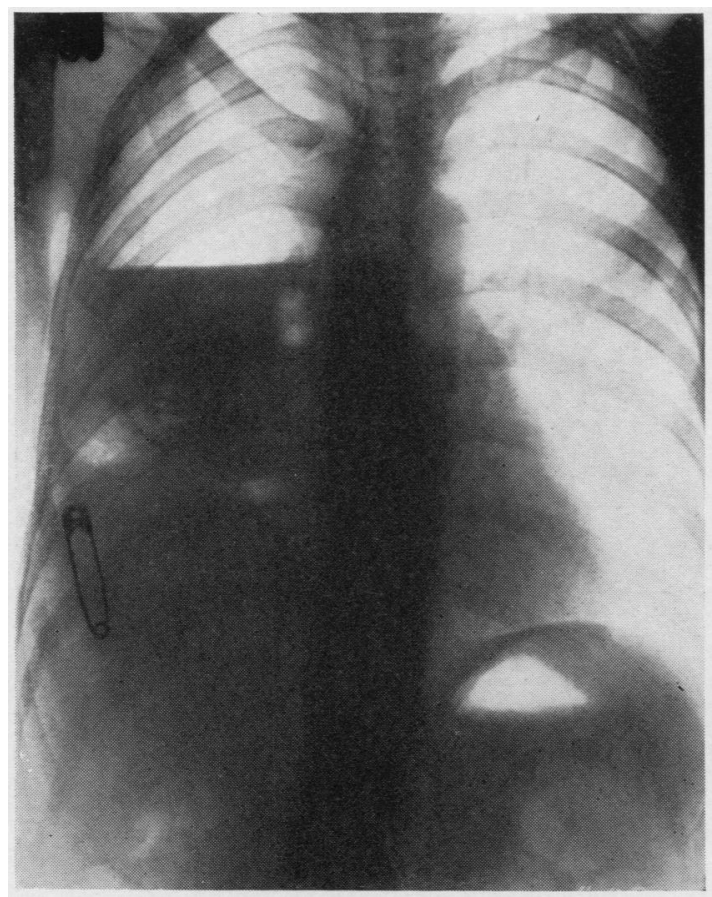

FIG. 20.-Empyema and subphrenic abscess following perforation of a duodenal ulcer. The lung required decortication.

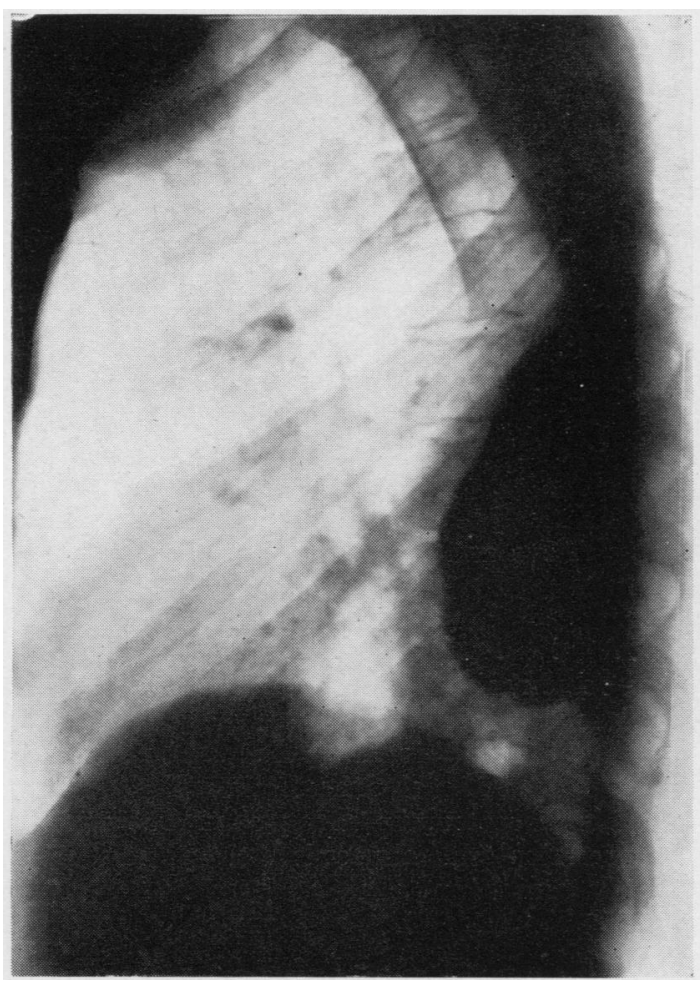

FIG. 21.-If the lung becomes adherent to the diaphragm, an empyema may form at a higher level in the paravertebral sulcus. The empyema has been located and "lipiodol" has been introduced before draining it so as to determine its extent.

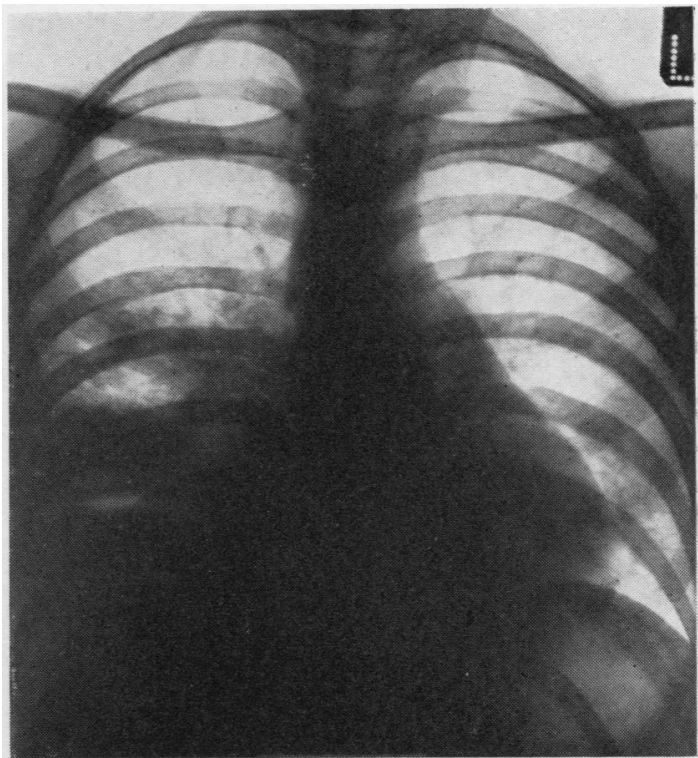

FIG. 22

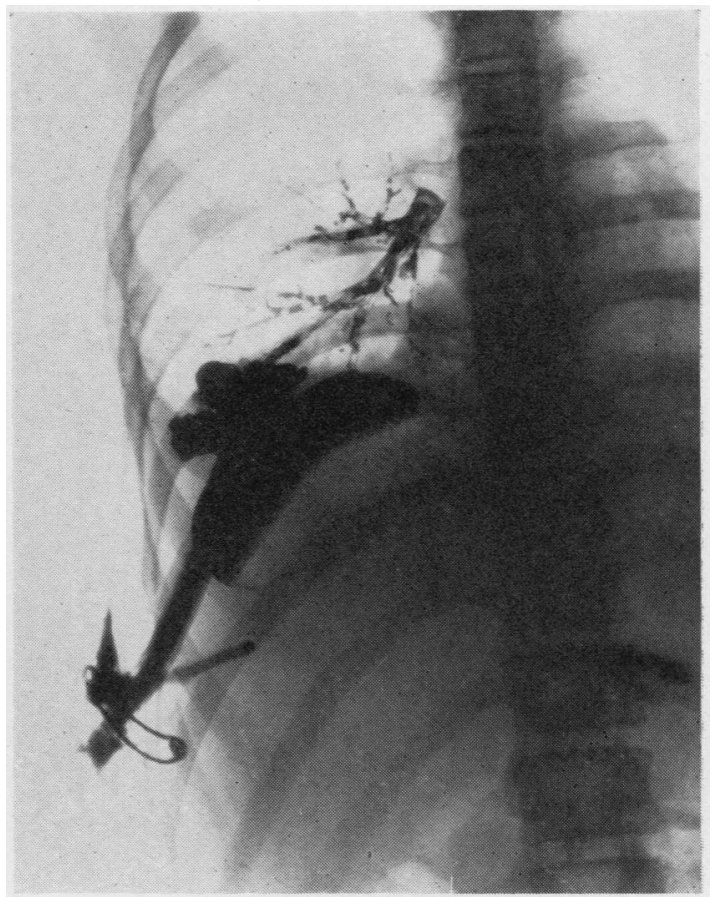

FIG. 23

FIGS. 22 and 23.-Right suprahepatic subphrenic abscess with bronchia I fistula. The straight radiograph shows an air space under the right dome of the diaphragm, which is elevated. There is a marked inflammatory reaction in the right lower lobe. Lipiodol was later introduced into the subphrenic abscess after drainage, and shows the bronchial fistula. 


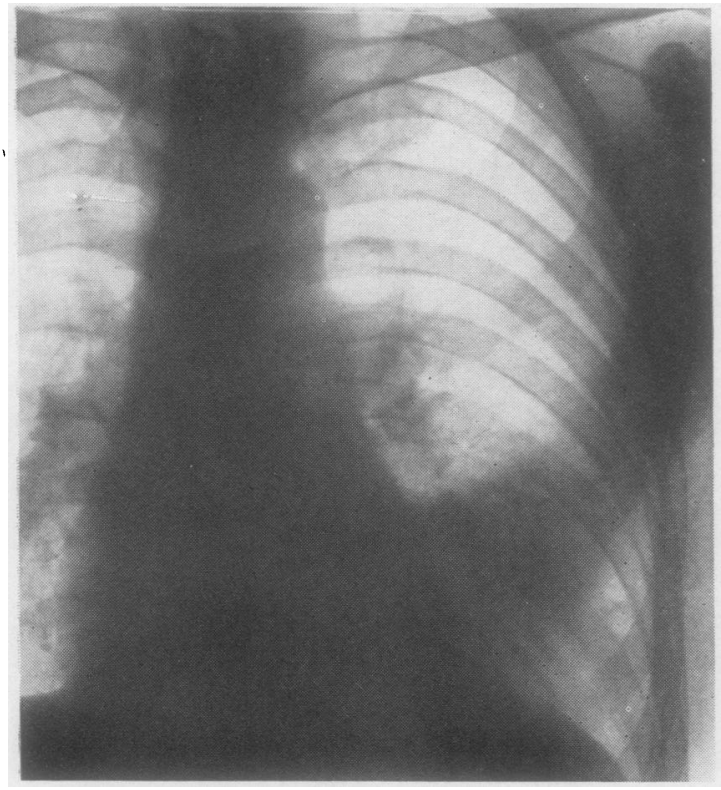

FIo. 24

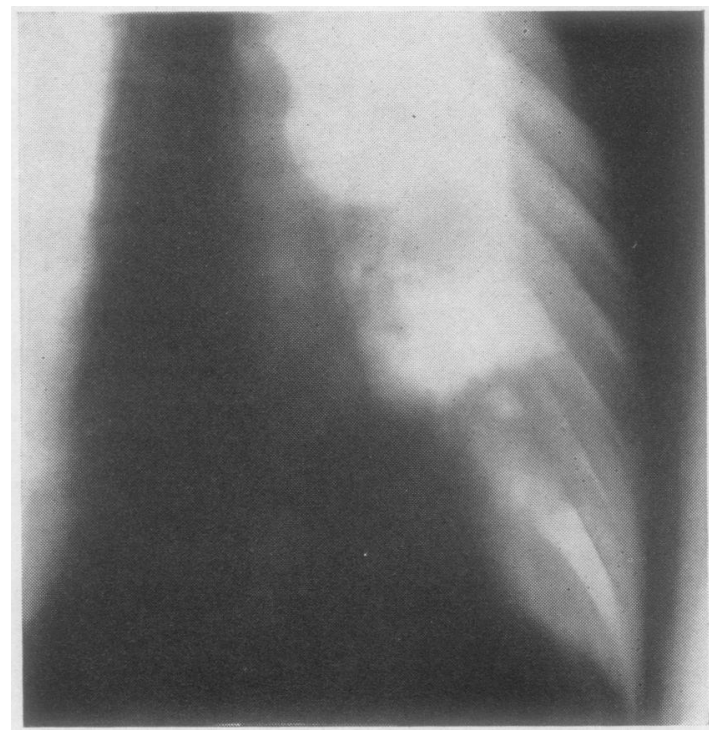

Fig. 25

FIGS. 24 and 25.-Left subphrenic abscess with bronchial fistula which presented as a bronchial neoplasm. The abscess was in the left posterior infrahepatic space (i.e., the lesser sac) and had ruptured into the lower lobe.

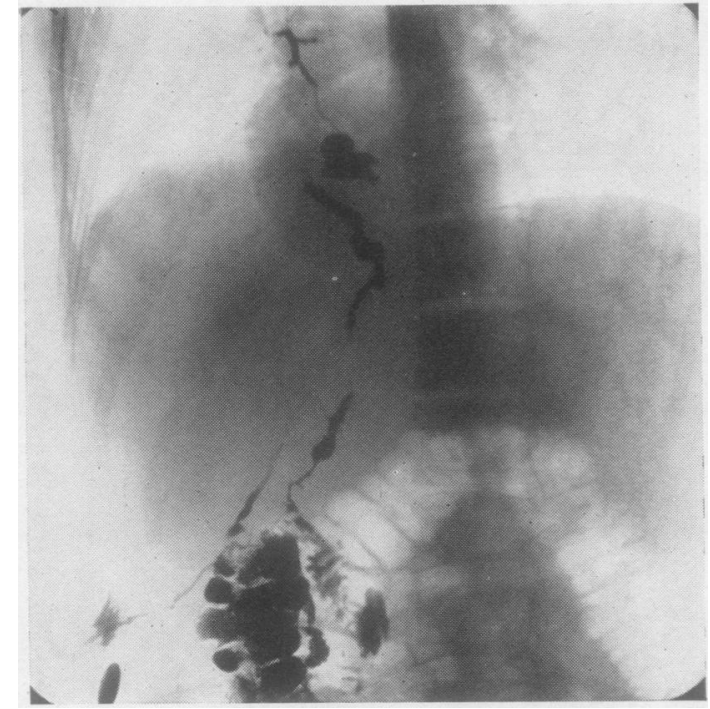

FIG. 26. - An abdominal wound began to discharge after a Polya type of gastrectomy, due to leakage from the duodenal stump. Lipiodol was injected into the sinus which demonstrated a track leading to the duodenal stump, where it divided into two. One passed down and entered the colon. The other went behind the liver into a small subphrenic abscess which had ruptured through the diaphragm into a lower lobe bronchus. be reached with a needle. When the diaphragm is elevated it soon becomes adherent to the chest wall and aspiration may be more safely performed without fear either of damaging the lung or of infecting the pleura.

The abdominal surgeon may decide to do a laparotomy in order to convince himself of the state of the subphrenic spaces. This may be performed through an upper paramedian or a subcostal incision. The posterior subphrenic spaces lie a long way from the anterior abdominal wall. When dealing with an obese adult, the arm must enter the abdomen as far as the elbow in order to palpate the posterior limits of the subphrenic spaces above and below the liver (Fig. 27).

\section{Surgical Treatment}

A subphrenic abscess cannot be treated by aspiration; it must be properly drained as soon as it has been diagnosed. This may be done comfortably under local anaesthesia; if a bronchial fistula is suspected, the patient should be placed on the operating table almost in a sitting position, for if he is turned on to his good side in the lateral position he may infect both lungs.

After considering the available clinical and radiological evidence I nearly always try to 
aspirate the abscess before making an incision. If I withdraw pus I make a small incision, and if not I make a big one.

Anterior abscesses between the liver and the diaphragm (left and right anterior suprahepatic), or between the liver and the stomach on the left side (left anterior infrahepatic), may be drained through a type of Kocher's incision.

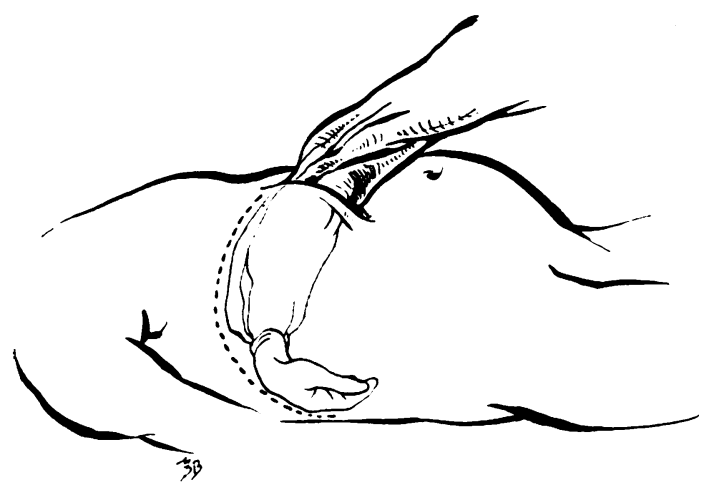

FIG. 27.-The posterior limits of the subphrenic spaces are a long way from the anterior abdominal wall. In an adult patient, through a subcostal incision, the arm needs to enter the abdomen as far as the elbow in order to palpate the spaces properly.

Rutherford Morison's pouch and the right infrahepatic space may be drained through the right loin. The lesser sac can be drained through the left loin.

The main suprahepatic spaces require a transthoracic approach by resecting the tenth, sometimes the ninth, rib. When in doubt I remove the rib from its angle to costal cartilage. The periosteum is then incised and frequently some fluid will be seen in the underlying pleural cavity. The pleura can be mobilized by dividing its attachments in the costophrenic angle; these consist of

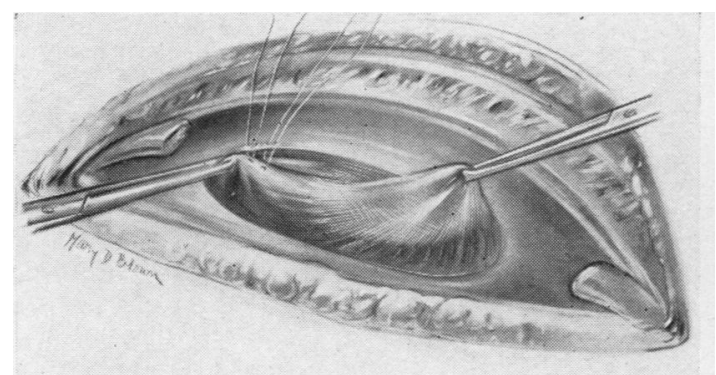

FIG. 28.-The ninth rib has been resected, the pleura incised, and the diaphragm, grasped with forceps, is being stitched to the parietal pleura and the intercostal muscles. small fibrous bands attached to the periosteum of each of the ribs and correspond to the attachment of the diaphragm.

If mobilization of the pleura is not possible, the parietal pleura may be pushed against the diaphragm with the left hand and the two stitched together; or the pleura may be opened and the diaphragm immediately grasped with tissue forceps and pulled through the hole in the pleura to obstruct it and the two stitched together (Fig. 28).

The diaphragm is opened, the pus evacuated, and the shape and extent of the abscess cavity seen and palpated. The wound is closed round a large soft rubber tube which is connected to an underwater drainage bottle. A post-operative radiograph is taken, and, if either air or fluid is present in the pleural cavity, it is aspirated and removed completely.

An empyema should be drained by a separate tube connected to another underwater seal bottle. The track of the empyema drainage tube must be separate from the one draining the subphrenic abscess. This is achieved by suturing diaphragm to chest wall between the two tubes and also

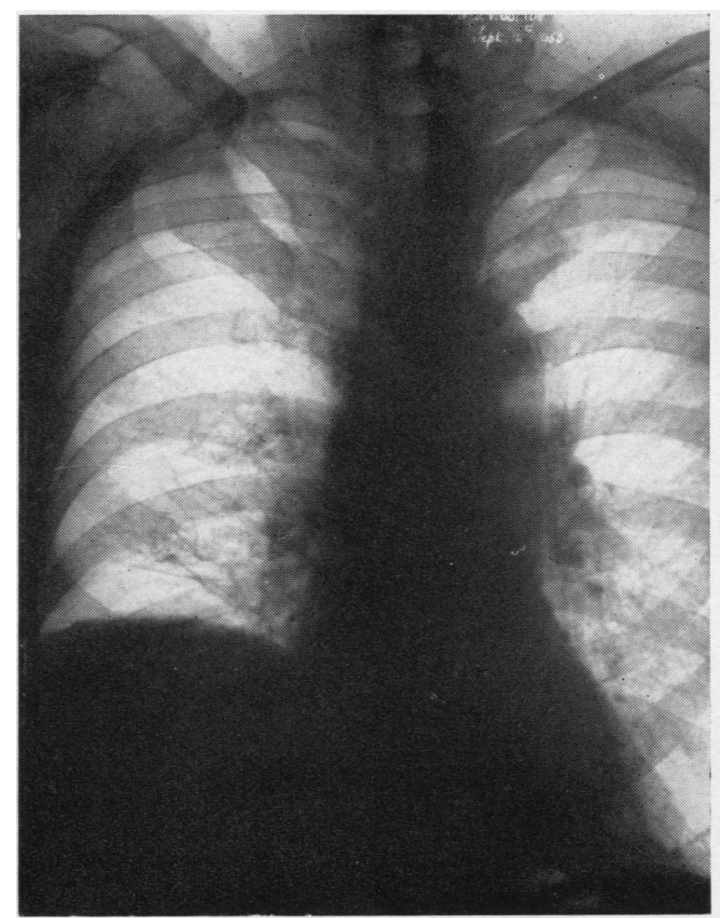

FIG. 29.-After a subphrenic abscess has healed, the diaphragm may remain elevated and never regain full movement, due to its becoming adherent to the chest wall. This radiograph was taken two years after healing of a subphrenic abscess. 
closing the wound and skin together between them. The subphrenic cavity will close first ; its walls are soft and mobile and they rarely take longer than a few days to heal (Fig. 29).

If there is a bronchial fistula present, the lung will be found to be adherent to the diaphragm. Drainage of the subphrenic abscess is sufficient and the fistula will close on its own. I have on one or two occasions mobilized the lung and sutured the fistula, but I do not think it was really necessary. After drainage of a subphrenic abscess with a bronchial fistula, bronchoscopy may be performed to prevent lung atelectasis and purulent bronchopneumonia.

In fact, after drainage of any subphrenic abscess, a watch must be kept radiologically on the clearing and re-expansion of the corresponding lower lobe. If it is not satisfactory, bronchoscopy should be carried out.

Two patients with small subphrenic abscesses healed spontaneously after the abscesses had ruptured into a bronchus and the contents been coughed up. Bronchial drainage of subphrenic abscess should be avoided by draining the abscess before this complication occurs. Three others required lobectomy at the same time as the sub- phrenic abscess was drained, because rupture of the abscess had destroyed the corresponding lower lobe.

\section{SUMMARY}

The anatomy of the subphrenic spaces has been described again with an attempt not to introduce a new nomenclature. Attention has been drawn to the size of the right suprahepatic space, and how pus localizes in either the anterior or posterior part of this space (both these parts are anterior to the right triangular ligament).

A brief description is given of 50 cases of subphrenic abscess treated in the Department of Thoracic Surgery at Leeds.

My thanks are due to Professor A. J. S. Johnstone, who investigated most of these patients radiologically, and to Professor P. R. Allison and Mr. J. A. Aylwin, whose patients I have included in this series. I wish also to thank Dr. Maxwell Telling for his advice and helpful criticism.

\section{REFERENCES}

Barnard, H. L. (1908). Brit. med. J., 1, 371, 429.

Harley, H. R. S. (1949). Thorax, 4, 1.

Mitchell, G. A. G. (1940). Brit. J. Surg., 28, 291.

Ochsner, A., and De Bakey, M. (1938). Int. Abstr. Surg., 66, 426.

Thorek, P. (1947). Surgery, 21, 739. 